\title{
David Cameron et la campagne du « Remain »
}

David Cameron and the Remain Campaign

\section{Pauline Schnapper}

\section{(2) OpenEdition}

\section{Journals}

Édition électronique

URL : http://journals.openedition.org/rfcb/1363

DOI : $10.4000 /$ rfcb. 1363

ISSN : 2429-4373

Éditeur

CRECIB - Centre de recherche et d'études en civilisation britannique

\section{Référence électronique}

Pauline Schnapper, «David Cameron et la campagne du « Remain » ", Revue Française de Civilisation Britannique [En ligne], XXII-2 | 2017, mis en ligne le 30 mai 2017, consulté le 19 avril 2019. URL : http:// journals.openedition.org/rfcb/1363; DOI : 10.4000/rfcb.1363

Ce document a été généré automatiquement le 19 avril 2019

Revue française de civilisation britannique est mis à disposition selon les termes de la licence Creative Commons Attribution - Pas d'Utilisation Commerciale - Pas de Modification 4.0 International. 


\section{David Cameron et la campagne $d u$ « Remain »}

David Cameron and the Remain Campaign

Pauline Schnapper

Our campaign was based on the simple proposition

that electorates don't vote against their own

pockets $^{1}$.

\section{Introduction}

1 Le choix de Cameron de promettre un référendum lors de son discours de Bloomberg le 23 janvier 2013 avait été dicté principalement par des considérations de politique intérieure ${ }^{2}$. Il s'agissait de répondre aux pressions exercées par le UKIP, par une partie de la presse (notamment le Daily Express et le Daily Mail) et par nombre de ses propres députés. Ceux-ci exigeaient un tel scrutin au motif que les électeurs britanniques n'avaient pas été consultés depuis 1975, alors même que l'Union européenne avait considérablement accru son domaine d'action et son influence sur les institutions du pays. Il avait aussi estimé qu'obtenir l'aval du peuple pour un maintien dans l'UE était devenu inévitable après le refus de Gordon Brown de ratifier le traité de Lisbonne par voie référendaire en 2007 et son propre recul sur la promesse faite alors de revenir sur cette ratification en cas de retour au pouvoir.

2 Le résultat du référendum sur l'Union européenne (UE), avec presque $52 \%$ des voix favorables au Brexit, a donc représenté un échec pour David Cameron qui avait pensé, en promettant un tel scrutin, satisfaire ses députés eurosceptiques et renforcer son leadership dans le parti tout en gardant son pays dans l'UE. Le Premier Ministre n'avait pourtant pas ménagé ses efforts, d'abord pour obtenir dans ses négociations à Bruxelles un accord qui pourrait satisfaire les députés hésitants, puis en se mettant en première ligne dans la campagne, du moins à partir de l'accord trouvé au Conseil européen des 18 et 19 février 2016. 
3 Il s'agit à présent de comprendre pourquoi cette stratégie a échoué et pourquoi, plus généralement, la campagne Britain Stronger in Europe n'a pas réussi à convaincre une majorité d'électeurs que le pays avait intérêt à rester dans l'organisation européenne dont elle est membre depuis 1973. Trois types d'explications peuvent être avancées, qui vont du plus général - la sous-estimation par Cameron du rejet de l'Europe dans son pays - aux plus tactiques, c'est-à-dire les erreurs commises pendant la campagne, en passant par les difficultés liées au contexte politique et partisan. Nous allons les examiner ici un par un.

\section{Un contexte général peu favorable et sous-estimé}

4 On ne peut qu'être frappé par la sous-estimation par Cameron de la vigueur du sentiment eurosceptique dans son pays (auquel il avait lui-même contribué pendant des années) et la surestimation de sa propre capacité à renverser en quelques mois, voire quelques semaines, une culture politique qui s'est consolidée dans son pays. En effet, autant la campagne du Remain s'est organisée à l'automne 2015, autant la campagne du Leave a bénéficié de réseaux mis en place dès les années 1990, au moment du débat sur le traité de Maastricht, à la fois dans les rangs conservateurs au Parlement et au sein d'organisations diverses, encore nombreuses dans les années 2000-20103. Ils pouvaient s'appuyer sur un climat général extrêmement critique vis-à-vis de l'Union européenne, souvent accusée dans le débat public d'être trop bureaucratique, non démocratique et de coûter trop cher, sans que s'élèvent beaucoup de voix pour la défendre ${ }^{4}$.

Cet euroscepticisme se retrouve à différents niveaux. Au sein des partis d'abord, en particulier le parti conservateur, travaillé depuis des années par un double courant, favorable à un retrait de l'UE pour le premier (hard eurosceptic), pour le moins critique pour une majorité de députés (soft eurosceptics), surtout depuis leur passage dans l'opposition entre 1997 et 2010. C'est à cette catégorie que Cameron disait appartenir, tout en donnant des gages aux premiers lorsqu'il a fait sortir ses députés européens du Parti Populaire Européen (PPE) et posé son "veto» au traité de consolidation fiscale européen en décembre 2011 (dont il n'avait pu empêcher l'entrée en vigueur) ${ }^{5}$. Il espérait, dans le même temps, réduire l'importance de la question européenne dans les débats internes du parti ${ }^{6}$.

Du côté travailliste, l'euroscepticisme des années 1960-1970 avait donné lieu à partir de la fin des années 1980, sous Neil Kinnock, John Smith et surtout Tony Blair, à un réalignement sur une position pro-européenne, suivi par l'immense majorité des élus avec quelques exceptions, parmi lesquelles le leader actuel Jeremy Corbyn, qui a soutenu du bout des lèvres le maintien dans l'UE. Dès la fin des années Blair pourtant, le discours travailliste, comme d'ailleurs celui des Libéraux-démocrates, se faisait plus critique sur le fonctionnement de l'Union européenne. Ainsi en 2007 Gordon Brown, devenu Premier Ministre, signait en catimini le traité de Lisbonne pour éviter une couverture médiatique critique au Royaume-Uni. Cette évolution reflétait la perception d'un durcissement de l'opinion publique sur le sujet.'

7 En effet, l'opinion publique au Royaume-Uni est toujours restée plus critique vis-à-vis de l'UE que dans les autres pays-membres, selon les chiffres compilés année après année par l'Eurobaromètre ${ }^{8}$. En dehors d'une courte période entre le milieu des années 1980 et le début des années 1990, il y a toujours eu une minorité non négligeable d'électeurs 
favorables à une sortie de l'UE (entre $20 \%$ et $40 \%$ selon les périodes). En 2015, seulement $32 \%$ des Britanniques interrogés avaient une opinion positive de l'Union européenne, contre une moyenne de $41 \%$ sur le continent. Seules la Grèce, Chypre et l'Autriche connaissaient des chiffres encore inférieurs ${ }^{9}$. Une vision utilitariste de l'UE a prévalu, qui ne s'est jamais accompagnée d'un sentiment d'appartenance ni d'une identification à une identité européenne commune. En 2015 toujours, 52\% des Britanniques interrogés déclaraient se sentir partiellement ou complètement des citoyens européens, contre une moyenne de $67 \%$ pour l'ensemble de l'Union ${ }^{10}$. La même année, près de la moitié des personnes interrogées dans une enquête fondée sur les données de British Social Attitudes indiquaient qu'elles considéraient l'Europe comme une menace pour l'identité britannique ${ }^{11}$.

Il n'est guère surprenant alors que les médias britanniques, et en particulier la presse nationale, aient reflété et encouragé cet euroscepticisme. Depuis des années, la couverture par les quotidiens nationaux des questions européennes est largement hostile, à l'exception du Guardian, de l'Independent (dont la version papier a maintenant disparu) et du Mirror ${ }^{12}$. Une des grandes différences entre le référendum de 1975 et celui de 2016 est qu'à l'époque la presse était très majoritairement favorable, à l'exception déjà du Daily Express, au maintien dans la CEE, tandis que cette fois-ci le Telegraph, le Sun, le Daily Star et le Daily Mail ont rejoint le camp du Leave. En prenant en compte les ventes des différents journaux nationaux, ce sont $80 \%$ des lecteurs qui lisaient en 2015-2016 des journaux favorables à la sortie de l'Union européenne ${ }^{13}$. Au niveau des articles sur le référendum, une majorité, tous journaux confondus, y était aussi favorables et les personnes citées plus souvent celles du camp du Leave ${ }^{14}$. Cet état de fait a lourdement pesé sur la campagne du Remain, ce dont témoigne Craig Oliver, qui montre une organisation obsédée par la façon dont les médias, y compris la BBC soumise au devoir d'impartialité, relayaient la propagande adverse: "There is almost nothing as formidable as the campaigning power of the right-wing press. As Ameet puts it, "We're discovering what it's like to be Ed Miliband' » (en référence à la façon dont l'ancien leader du parti travailliste avait été éreinté par la presse) ${ }^{15}$.

Un autre facteur de nature contextuelle doit aussi être pris en considération. Il s'agit de l'impact des politiques d'austérité mises en place par le gouvernement de coalition à partir de 2010, qui a eu des conséquences, sinon sur l'emploi, en tout cas sur les revenus et sur les services publics locaux. En d'autres termes, les effets généraux de la mondialisation, y compris les pertes d'emplois dans les secteurs industriels traditionnels, n'ont pas été compensés pendant cette période par un engagement de l'Etat, bien au contraire. Le vote pour sortir de l'UE a clairement été plus important dans les zones les plus désavantagées sur le plan socio-économique, qui sont aussi celles où les réductions budgétaires ont été les plus violentes et où le vote pour le UKIP a été le plus élevé aux élections européennes de 2014. Dans leur étude, Becker, Fetzer et Novy en concluent qu'une réduction plus limitée des dépenses publiques entre 2010 et 2015 aurait peut-être pu inverser le résultat du référendum, ce qui est impossible à prouver mais donne une idée de l'ampleur des effets de cette politique ${ }^{16}$.

Par ailleurs, ce n'est pas seulement le climat économique et politique intérieur qui était peu propice à un tel scrutin, mais aussi la situation européenne dans son ensemble. Au moment du référendum britannique, l'UE était encore confrontée à plusieurs crises graves et concomitantes. La crise de la zone euro, qui n'avait jamais vraiment été réglée, avait contribué à l'idée largement répandue outre-Manche que l'UE n'était pas ou plus 
capable d'assurer la prospérité économique de ses Etats membres, ce qui avait été un argument important pour justifier l'entrée dans la CEE à l'origine. Il s'y ajoutait depuis l'été 2015 la crise des réfugiés qui avait provoqué, entre autres, de graves tensions entre Etats-membres du nord et du sud de l'Europe, ainsi qu'avec les pays de l'Est, Hongrie, Pologne et Slovaquie particulièrement. Les attaques terroristes en France et en Belgique représentaient une crise sécuritaire intérieure qui se cumulait avec les crises extérieures, en Irak/Syrie et avec la Russie depuis 2014. Enfin, à ces crises d'origine exogène s'ajoutait une crise politique intérieure, avec la perte de confiance et le rejet croissant par les opinions publiques européennes du projet supranational, reflété dans les sondages, les résultats des élections européennes (qui ont permis aux partis populistes et antieuropéens d'être de plus en plus représentés au Parlement) et les résultats des récents référendums. Les rejets français et néerlandais du traité constitutionnel en 2005 , le rejet du traité de Lisbonne par les électeurs irlandais en 2008 et le rejet par les Danois de la participation au volet Justice et Affaires intérieures de l'UE en 2015 auguraient mal d'un vote positif outre-Manche tant était grand le discrédit des institutions européennes.

11 Dans un tel contexte, il était optimiste de la part de David Cameron de penser qu'il pourrait renverser ce climat anti-européen en quatre mois et convaincre une majorité de ses compatriotes des bienfaits relatifs de l'Union européenne. On peut expliquer son espoir par les deux succès électoraux remportés par son parti et lui-même en Ecosse en 2014 et aux élections législatives de 2015. Lors de la campagne pour le référendum sur l'indépendance écossaise, il avait finalement réussi, avec la campagne transpartisane Better Together, à obtenir une majorité de $55 \%$ pour le maintien dans le Royaume-Uni malgré une très forte poussée du mouvement indépendantiste mené par le SNP et des sondages qui donnaient les deux camps au coude-à-coude jusqu'à quelques jours avant le scrutin. En 2015, Cameron et le parti conservateur avaient, contre toute attente, battu les travaillistes et obtenu une majorité absolue des sièges à la Chambre des Communes grâce, en particulier, à l'efficacité de sa campagne personnelle. Le Premier Ministre pouvait imaginer alors réussir la même performance un an plus tard dans ce référendum en utilisant de nouveau les recettes de son gourou australien, Lynton Crosby, pour qui il suffisait de marteler un message simple sur l'économie pour l'emporter ${ }^{17}$.

\section{Une équation partisane complexe}

12 Le camp du Remain ne s'est pas seulement heurté à une culture politique imprégnée d'euroscepticisme depuis des années. Il a été également fragilisé par la nature même d'une campagne transpartisane dont les différentes composantes étaient elles-mêmes divisées (comme c'était d'ailleurs le cas en 1975).

13 La stratégie de Cameron était d'abord destinée à son propre parti: il s'agissait, après avoir concédé l'organisation d'un référendum, d'obtenir un accord de renégociation à Bruxelles qui satisferait sinon les anti-européens irréductibles, en tout cas le "marais » de députés conservateurs qui attendaient une réforme significative de l'UE et de la position britannique dans cette union. Une fois ceux-ci convaincus, la victoire suivrait lors du scrutin qui serait organisé rapidement, pendant que la popularité du Premier Ministre était encore élevée.

Ce scénario ne s'est pas réalisé. Lorsque Cameron est rentré du Conseil européen de février 2016 avec un accord comprenant notamment des garanties pour les non-membres de la zone euro et l'instauration d'un emergency brake (frein de secours) permettant au 
gouvernement britannique de bloquer l'accès des immigrants européens à certaines prestations sociales pendant sept ans, la presse eurosceptique et certains de ses collègues lui reprochèrent immédiatement la faiblesse du résultat. Le Daily Mail titra: «Call That a Deal, Dave ?» Michael Gove et quatre autres membres du Cabinet (Chris Grayling, Priti Patel, John Wittingdale et Theresa Villiers) profitèrent de la suspension de la règle de la responsabilité collective qui leur avait été accordée par le Premier Ministre pour annoncer aussitôt qu'ils rejoignaient le camp du Leave. Plus grave pour la campagne, l'ancien maire de Londres, Boris Johnson, très populaire auprès de la base du parti, les rejoignit au bout de trois jours. Au lieu de la soixantaine de députés escomptés, Cameron dut finalement faire face à la fronde de 138 élus conservateurs à la Chambre des Communes, soit plus du tiers du groupe parlementaire ${ }^{18}$.

Les divisions du parti conservateur et l'espoir de Cameron de rester à la tête d'un parti réunifié après le référendum eurent des conséquences directes sur le déroulement de la campagne Stronger In et sa couverture médiatique. Cameron, voulant limiter les attaques blue on blue, s'opposa ainsi à toute attaque directe contre les leaders conservateurs du camp adverse pour ne pas alimenter les divisions au sein de son parti. Craig Oliver cite un exemple datant de mars 2016:

Stronger In is pushing hard to send round social media interventions about Iain Duncan Smith, essentially claiming he's a hypocrite, who was prepared to use dodgy stats in something completely unrelated last year. I call Will Straw, admitting I don't want to wind this up on the Tory side, but more importantly we can't give them examples of us slinging mud. We have to be the sensible people who rise above it, no matter what the provocation ${ }^{19}$.

16 Un poster représentant Boris Johnson dans la poche de Nigel Farage, rappelant celui de la campagne de 2015 montrant Ed Miliband dans la poche d'Alex Salmond, fut ainsi retiré au dernier moment ${ }^{20}$. Pour la même raison, l'équipe de Cameron refusa un débat télévisé avec Boris Johnson ou Michael Gove ${ }^{21}$. Les médias, pour leur part, consacrèrent une grande part de leur couverture au conflit interne au parti, en particulier au duel entre Cameron et Johnson, au risque de décourager les électeurs travaillistes d'aller voter ${ }^{22}$. Ils évoquèrent beaucoup moins les divisions, pourtant bien réelles aussi, au sein du camp favorable au Brexit entre les partisans d'une réduction drastique de l'immigration, soutiens du UKIP généralement, et les partisans du libre échange intégral en dehors de l'UE, souvent conservateurs, pour qui la question migratoire était secondaire.

Il s'ajoutait à ce souci de protéger l'unité du parti conservateur un autre lié au fait de partager la campagne avec d'autres partis, notamment les travaillistes. Comme l'écrivait Craig Oliver le 28 mai :

I'm beginning to realise - the strength of our campaign that we celebrate (a broad coalition taking in most of the government, Labour, the Lib Dems, the Greens and the TUC), is also a profound weakness. We can't exercise the fingertip control that we had over messaging in the general election ${ }^{23}$.

18 Si la cohabitation n'a pas posé de problème au sein de l'organisation Britain Stronger in Europe, co-dirigée par Will Straw (parti travailliste) et Craig Oliver (responsable de la communication au 10 Downing Street, officiellement nommé fin mai), il n'en est pas allé de même pour les apparitions médiatiques. Non seulement Jeremy Corbyn a fait campagne du bout des lèvres en faveur du maintien dans l'UE, mais à aucun moment il n'a accepté de faire campagne avec David Cameron, ni d'ailleurs avec son prédécesseur à la tête du parti, Tony Blair ${ }^{24}$. Au-delà des divergences idéologiques, il s'agissait dans son esprit de ne pas rééditer l' « erreur » de la campagne unioniste en Ecosse, qui avait réuni 
Conservateurs, travaillistes et Libéraux-démocrates et finalement abouti à la défaite retentissante des travaillistes aux élections de 2015 en Ecosse, écrasés par le SNP ${ }^{25}$. Le résultat de ce choix fut qu'à quelques interventions près (par exemple la présence concomitante de David Cameron, Neil Kinnock et Paddy Ashdown dans les locaux de la campagne pour appeler des électeurs), Stronger In ne put pas présenter un front uni aux électeurs et la campagne travailliste, dirigée par Alan Johnson, fut quasiment inaudible en l'absence du leader du parti ${ }^{26}$. Craig Oliver, dans son journal de la campagne, ne cesse de se plaindre du chaos régnant dans le parti travailliste et de son inefficacité dans la campagne ${ }^{27}$. A l'inverse, une des rares députées travaillistes favorable au Leave, Gisela Stuart, fut très présente dans les médias et participa à plusieurs débats à la radio et à la télévision, alimentant l'impression que son parti était, lui aussi, divisé sur la question européenne. Il n'est pas étonnant dès lors que beaucoup d'électeurs travaillistes n'aient pas été sûrs de la position de leur parti sur la question posée, ce qui a affaibli la campagne Stronger In.

19 A la décharge des partisans du Remain travaillistes, les principaux thèmes de la campagne référendaire, l'économie et l'immigration, mettaient le parti dans une position difficile. Après 2010, les travaillistes ont eu beaucoup de mal à se faire entendre sur les questions économiques, car les Conservateurs sont parvenus à convaincre de nombreux électeurs de leur responsabilité dans la crise financière de 2008. Surtout, les travaillistes n'avaient pas de position commune sur l'immigration, sujet délicat depuis la décision de Tony Blair d'ouvrir en 2004 les frontières aux ressortissants des nouveaux Etats-membres de l'Europe de l'Est, conduisant à une arrivée massive de migrants dans les années qui suivirent ${ }^{28}$. Devant les réactions très négatives d'une part de leur électorat, deux attitudes très différentes se sont exprimées: d'un côté Jeremy Corbyn, leader depuis 2015, défendait paradoxalement cette politique en expliquant que l'immigration produisait des effets positifs sur l'économie et que les problèmes des Britanniques venaient non des immigrés mais des réductions budgétaires appliquées depuis 2010. De l'autre, beaucoup de députés souhaitaient au contraire limiter l'entrée des immigrants européens dans l'espoir de reconquérir la partie de leur électorat tentée par le Brexit (et le vote UKIP plus généralement).

Enfin, Cameron n'a pas réellement pu compter non plus sur le soutien des Libérauxdémocrates, très affaiblis par leur défaite catastrophique aux élections législatives, qui n'a laissé que huit sièges au parti à la Chambre des Communes. La visibilité médiatique des Lib Dems pendant la campagne référendaire a été des plus limitées, son leader Tim Farron n'apparaissant dans aucun des débats télévisés programmés pendant cette période 29 .

\section{Des erreurs tactiques}

21 Au-delà des causes structurelles examinées ci-dessus, la campagne du Remain a aussi souffert de facteurs conjoncturels qui lui étaient peu favorables, dont certains étaient le résultat de la situation évoquée plus haut.

Tout d'abord, l'entrée en campagne de David Cameron a été tardive, puisqu'il ne s'est clairement engagé pour le maintien dans l'UE qu'après le Conseil européen de février 2016. C'était là le résultat d'une tactique dans laquelle le Premier Ministre s'est enferré, consistant à prétendre que sa position sur l'appartenance à l'Europe dépendrait 
uniquement du résultat de la renégociation à Bruxelles, laissant entendre qu'il pourrait même être favorable à une sortie de l'UE s'il n'obtenait pas satisfaction. Cela laissait évidemment entendre qu'en l'état, l'appartenance à l'UE n'était pas forcément favorable au Royaume-Uni, et que seule une réforme profonde de son fonctionnement pourrait la rendre positive. Cette posture n'a trompé personne mais a empêché le Premier Ministre de prendre clairement parti pendant plusieurs mois et de développer un contre-discours favorable à l'Europe. L'argument utilisé à plusieurs reprises par Craig Oliver pour justifier cette approche est qu'en se présentant comme agnostique sur la question au départ, Cameron pourrait rallier à lui les indécis ${ }^{30}$. Mais dans le même temps, il a finalement très peu défendu et utilisé le résultat de sa négociation comme argument pendant la courte campagne qui a suivi. Celle-ci a donc été doublement affaiblie par cette tactique.

Sous la pression des Brexiteers, David Cameron dût aussi accorder la règle à partir du 27 mai dite du Purdah, qui veut que les fonctionnaires des ministères (mais aussi le gouverneur de la Banque d'Angleterre) ne puissent aider le gouvernement pendant le mois qui précède une élection ${ }^{31}$. Concrètement, cela signifiait qu'à partir de là le gouvernement ne pouvait pas dépenser d'argent pour la campagne ni communiquer sur les bénéfices de l'appartenance à l'UE et les risques d'un éventuel Brexit, laissant seule l'organisation Stronger In.

Le troisième problème rencontré par la campagne a déjà été évoqué rapidement. Deux grands thèmes l'ont dominée: l'économie et l'immigration, rassemblées sous la question générale de la souveraineté. La campagne du Remain était en position de force sur les conséquences économiques négatives d'un éventuel Brexit, soutenue par les analyses de nombreuses institutions internationales (FMI, Banque Mondiale, OCDE) et même par le président Obama, qui déclara à Londres qu'un Royaume-Uni isolé ne serait pas prioritaire pour négocier un accord de libre échange avec les Etats-Unis ${ }^{32}$. Surnommée « Project Fear " par ses détracteurs, la tactique consistant à concentrer l'argumentation sur l'économie a été réaffirmée pendant toute la campagne et considérée comme un succès, puisque les leaders de la campagne adverse n'ont pas convaincu de leur capacité à proposer une alternative crédible au marché unique. Mais la campagne du Leave a alors réorienté le débat sur l'immigration, avec beaucoup plus de succès. Stronger In a mis du temps à réagir à l'offensive adverse, qui citait la menace d'une entrée de la Turquie dans l'Union européenne et la nécessité de reprendre le contrôle des frontières nationales. La prise de conscience n'a eu lieu, d'après Craig Oliver, que fin avrili33. Même ensuite, le message de la campagne a continué à être axé sur les risques du Brexit pour l'économie, avec l'idée de jouer la rationalité contre les passions («Heads vs Hearts»), en pleine conscience que la campagne du Remain n'avait pas de message convaincant sur l'immigration, pour les raisons indiquées plus haut ${ }^{34}$. La cacophonie entre les principaux protagonistes de la campagne du In et au sein des partis a rendu impossible l'expression d'un message simple et clair comparable à celui de la campagne adverse, avec son "Take Back Control». Cameron a été constamment sur la défensive sur l'immigration et l'échec de sa politique sur la question lui a été rappelé avec la publication, au milieu de la campagne, des chiffres de l'immigration pour 2015, indiquant plus de 330,000 entrées légales, dont la moitié en provenance de l'Union européenne ${ }^{35}$.

25 La campagne a enfin été handicapée par la complexité de son message sur les avantages relatifs de l'Union européenne face à un discours anti-establishment simpliste. Nick Clegg raconte sa frustration à ce sujet: 
I suggested to Cameron that an equally simple but more rousing refrain - 'Keep Britain Safe' (from recession, from the break-up of the UK, from climate change, from cross-border crime, et cetera) - should be deployed in return [to 'Take Back Control']. He responded that he was more inclined to stick with their appeal to level-headed caution: 'Don't Risk It'. I did not pursue it further - not least because I believed, at the time, that the Remain camp would still win. In the end, of course, the heart won out over the head - not least because Britain's heart, when it comes to the $\mathrm{EU}$, has never fully been in it in the first place ${ }^{36}$.

Le type d'argumentation déployé pour convaincre les électeurs de rester dans l'Union européenne nécessitait de longs développements difficilement compatibles avec une campagne de terrain. Il était clairement moins mobilisateur que son concurrent. Le porteà-porte est resté par ailleurs limité, d'après Craig Oliver ${ }^{37}$. Sur le terrain, la campagne adverse, Grassroots Out, qui regroupait plusieurs organisations et supporters du UKIP, a été nettement plus présente.

\section{Conclusion}

La campagne Britain Stronger in the EU a pâti d'un ensemble de faiblesses et d'erreurs tactiques, dont certaines dépassaient la personne du Premier Ministre. Le climat général, marqué par la montée de l'euroscepticisme, mais aussi par un rejet des élites et un sentiment d'abandon économique et social dans beaucoup de milieux populaires, a alimenté le vote de rejet. L'organisation de la campagne, les incohérences entre les différents partis politiques impliqués, le manque d'engagement des travaillistes et le choix de thématiques qui n'ont finalement pas autant porté que prévu auprès de l'électorat expliquent le résultat du vote.

Pour autant, ces facteurs n'exonèrent pas David Cameron, qui s'est trouvé par la force des choses en première ligne dans cette campagne, de toute responsabilité. Même si lui et ses conseillers ont estimé qu'un référendum était politiquement inévitable, il était présomptueux de sa part d'imaginer le remporter facilement à partir d'un accord de renégociation forcément limité et qu'il a finalement peu utilisé. Une forme d'hubris l'a empêché de voir les obstacles structurels à une telle victoire, qui ne sont d'ailleurs plus propres à son pays mais affectent l'ensemble de l'UE. Le Premier Ministre a aussi payé, plus généralement, des années de discours et de pratiques (entre 2010 et 2015) hostiles à l'intégration européenne, destinés à satisfaire la frange la plus radicale de ses troupes, qui ont entretenu l'idée d'une irréductibilité entre identité britannique et européenne. Cette politique à courte vue s'est retournée contre lui et lui a finalement coûté sa carrière politique.

Pauline Schnapper est professeur de civilisation britannique à l'université de la Sorbonne Nouvelle Paris 3, auteur (avec David Baker) de Britain and the Crisis in the European Union, Palgrave, 2015.

D'Ancona, Matthew, "Brexit: how a fringe idea took hold of the Conservative Party", The Guardian, 15 juin 2016.

Insulting the Public? The British Press and the European Union ${ }^{\mathrm{e}}$

Baker, David et Schnapper, Pauline, Britain and the Crisis in the European Union, Basingstoke, Palgrave, 2015.

Becker, Sascha O., Fetzer, Thiemo et Novy, Dennis, "Who Voted for Brexit? A Comprehensive District-Level Analysis", Working Paper Series, Centre for Competitive 
Advantage in the Global Economy, University of Warwick, October 2016, http:// www2.warwick.ac.uk/fac/soc/economics/research/centres/cage/manage/ publications/305-2016_becker_fetzer_novy.pdf Referendum Analysis 2016, http://www.referendumanalysis.eu/eu-referendumanalysis-2016/section-6-parties/the-liberal-democrats-the-eu-referendums-invisibleparty/ and the Liberal Democrats since 2010" dans Tournier-Sol, Karine et Gifford, Chris (dir.), The UK Challenge to Europeanization: The Persistence of British Euroscepticism, Basingstoke, Palgrave, 2015, pp. 117-133. Opinion and Party Management", Government and Opposition, Vol. 37, No. 1, 2002, pp. 211-230. 


\section{NOTES}

1. Craig Oliver, Unleashing Demons : The Inside Story of Brexit, Londres, Hodder \& Stoughton, 2016, p. $10-11$.

2. L'auteur tient à remercier Anand Menon et David Baker pour leurs remarques sur cet article.

3. Agnès Alexandre-Collier, La Grande-Bretagne eurosceptique ? L'Europe dans le débat politique britannique, Editions du Temps, 2002; Simon Usherwood, "Opposition to the European Union in the UK: The Dilemma of Public Opinion and Party Management", Government and Opposition, Vol. 37, No. 1, 2002, pp. 211-230. Voir aussi Matthew d'Ancona, "Brexit: how a fringe idea took hold of the Conservative Party", The Guardian, 15 juin 2016 et l'article sur Daniel Hannan de Sam Knight, "The man who brought you Brexit", The Guardian, 29 septembre 2016.

4. Pauline Schnapper, "The Dilemma of Pro-European Parties in the UK : the Case of Labour and the Liberal Democrats since 2010" dans Karine Tournier-Sol et Chris Gifford (eds), The UK Challenge to Europeanization: The Persistence of British Euroscepticism, Basingstoke, Palgrave, 2015, pp. 117-133.

5. Anand Menon et John Paul Salter, "Brexit : initial reflections", International Affairs, vol. 92, n 6, 2016, à paraître.

6. , Philip Lynch et Richard Whitaker, "Where There is Discord, Can They Bring Harmony ? Managing Intra-Party Dissent on European Integration in the Conservative Party", British Journal of Politics and International Relations, vol. 15, n³, 2013, pp. 317-339; Philip Lynch, "Conservative modernisation and European integration: From silence to salience and schism", British Politics, vol. 10, n², 2015, pp. 185-203.

7. Pauline Schnapper, "The dilemma of pro-European parties...", op. cit.

8. David Baker et Pauline Schnapper, Britain and the Crisis in the European Union, Basingstoke, Palgrave, 2015, p. 86.

9. Enquête Eurobaromètre $n^{\circ}$ 83, printemps 2015, p. 9, disponible sur http://ec.europa.eu/ public_opinion/archives/eb/eb83/eb83_first_en.pdf, consulté le 3 octobre 2016.

10. Ibid., p. 28.

11. John Curtice, "How deeply does British euroscepticism run?", février 2015, disponible sur http://whatukthinks.org/eu/wp-content/uploads/2016/02/Analysis-paper-5-How-deeply-doesBritains-Euroscepticism-run.pdf, consulté le 3 octobre 2016.

12. Peter J. Anderson et Anthony Weymouth, Insulting the Public? The British Press and the European Union, Londres, Routledge, 1999 (2è éd.) : Oliver Daddow, "Playing Games with History: Tony Blair's European Policy in the Press", British Journal of Politics and International Relations, vol. 9, 2007, pp. 582-598.

13. http://www.referendumanalysis.eu/eu-referendum-analysis-2016/section-3-news/the-pressand-the-referendum-campaign/, consulté le 2 octobre 2016.

14. David A. L. Levy, Billur Aslan and Diego Bironzo, UK press coverage of the EU referendum (Oxford: University of Oxford/Reuters Institute for the Study of Journalism, Sept. 2016), http:// reutersinstitute.politics.ox.ac. uk/sites/default/files/UK\%20Press\%20Coverage\%20of\%20the\% 20EU\%20Referendum_0.pdf.

15. Craig Oliver, op. cit., p. 74. Voir aussi p. 242 à propos de la BBC.

16. Sascha O. Becker, Thiemo Fetzer et Dennis Novy, "Who Voted for Brexit? A Comprehensive District-Level Analysis", Working Paper Series, Centre for Competitive Advantage in the Global Economy, University of Warwick, October 2016, http://www2.warwick.ac.uk/fac/soc/economics/ 
research/centres/cage/manage/publications/305-2016_becker_fetzer_novy.pdf, consulté le 20 octobre 2016.

17. Tom McTague, Alex Spence et Edward-Isaac Dovere, "How David Cameron blew it: The behind-the-scenes story of a failed campaign to keep Britain in the European Union", Politico, 25 June 2016, http://www.politico.eu/article/how-david-cameron-lost-brexit-eu-referendumprime-minister-campaign-remain-boris-craig-oliver-jim-messina-obama/, consulté le 4 octobre 2016.

18. BBC, "EU vote: Where the Cabinet and other MPs stand", 22 June 2016, http://www.bbc.com/ news/uk-politics-eu-referendum-35616946.

19. Craig Oliver, Unleashing Demons, op. cit., p. 118-119.

20. Rafael Behr, "How remain failed : the inside story of a doomed campaign", The Long Read, The Guardian, 5 juillet 2016. Voir aussi Craig Oliver, Unleashing Demons, op. cit., p. 248.

21. Craig Oliver, Unleashing Demons, op. cit., p. 219.

22. David Deacon et al, "The narrow agenda : how the news media covered the Referendum", EU Referendum Analysis: Medias, Voters and the Campaign, http://www.referendumanalysis.eu/ eu-referendum-analysis-2016/section-3-news/the-narrow-agenda-how-the-news-mediacovered-the-referendum/, consulté le 5 octobre 2016.

23. Craig Oliver, Unleashing Demons, op. cit., p. 255.

24. "Jeremy Corbyn to urge 'warts and all' backing of European Union", The Guardian, 14 avril 2016.

25. Craig Oliver, Unleashing Demons, op. cit., p. 181.

26. Ibid., p. 158.

27. Ibid., pp. 304-305.

28. Thom Brooks, "The immigration debate : Labour versus Leave in the battle to win public trust", EU Referendum Analysis 2016, http://www.referendumanalysis.eu/eu-referendumanalysis-2016/section-6-parties/the-immigration-debate-labour-versus-leave-in-the-battle-towin-public-trust/, consulté le 6 octobre 2016.

29. Andrew Russell, “The Liberal Democrats: the EU Referendum's invisible party”, EU Referendum Analysis 2016, http://www.referendumanalysis.eu/eu-referendum-analysis-2016/ section-6-parties/the-liberal-democrats-the-eu-referendums-invisible-party/, consulté le 6 octobre 2016.

30. Voir par exemple Craig Oliver, Unleashing Demons, op. cit., p. 228 ou 310.

31. Pour plus de détails sur cette spécificité britannique, voir “ 'Purdah' before elections and referendums", Briefing Paper 05262, House of Commons Library, 26 mai 2016, http:// researchbriefings.parliament.uk/ResearchBriefing/Summary/SN05262\#fullreport, consulté le 25 octobre 2016.

32. "Barack Obama : Brexit would put UK 'back of the queue' for trade talks", The Guardian, 22 avril 2016.

33. Craig Oliver, Unleashing Demons, op. cit., p. 209.

34. Ibid., p. 284 et 322.

35. "Net migration to UK rises to 333,000 - second highest on record", BBC News, 26 mai 2016, http://www.bbc.com/news/uk-politics-eu-referendum-36382199, 6 octobre 2016.

36. Nick Clegg, Politics Between the Extremes, Londres, The Bodley Head, 2016, p. 197.

37. Craig Oliver, Unleashing Demons, op. cit., p. 251. 


\section{RÉSUMÉS}

Cet article étudie les raisons pour lesquelles la campagne pour rester dans l'Union européenne, menée par David Cameron, a échoué à convaincre une majorité d'électeurs britanniques lors du référendum du 23 juin 2016. Il aborde d'abord des raisons structurelles sous-estimées par le Premier Ministre, en particulier le climat général d'euroscepticisme au Royaume-Uni consolidé par les différentes crises rencontrées par l'UE depuis 2008. Ensuite il analyse les facteurs politiques qui ont entravé l'efficacité de la campagne et enfin les erreurs tactiques qui ont été commises au cours de la campagne, notamment l'impossibilité de présenter un message clair sur l'immigration, qui ont laissé le champ libre à la campagne du Leave.

This article analyses the reasons why the Remain campaign, led by David Cameron, failed to convince a majority of the British voters to stay in the European Union on the 23 June 2016. It first analyses the structural reasons underestimated by the Prime Minister, in particular the general eurosceptic climate in his country, strengthened by the multiple crises confronted by the EU since 2008. It then analyses the political factors which constrained the Remain campaign and finally the tactical mistakes made during the campaign, in particular the failure to articulate a clear message on immigration, which left the floor open to the Leave campaign.

INDEX

Mots-clés : Brexit, David Cameron, référendum, parti travailliste

Keywords : Brexit, David Cameron, referendum, Labour party

\section{AUTEUR}

\section{PAULINE SCHNAPPER}

Université Sorbonne Nouvelle Paris 3 